\title{
Appropriate Curriculum: Enabling the Student to Meet the Transdisciplinary Challenges of a Sustainable Society
}

\section{Helmut Burkhardt}

Ryerson University

\section{Please Cite:}

Burkhardt, H. (1991). Appropriate curriculum: Enabling the student to meet the transdisciplinary challenges of a sustainable society. Proceedings of the 1991 International Symposium on Technology and Society - ISTAS `91, 264-270. doi:10.1109/ISTAS.1991.700376

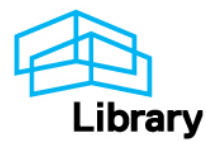




\title{
APPROPRIATE CURRICULUM: ENABLING THE STUDENT TO MEET THE TRANSDISCIPLINARY CHALLENGES OF A SUSTAINABLE SOCIETY
}

\author{
H. Burkhardt \\ Ryerson Polytechnical Institute \\ Toronto, Ontario, Canada \\ Presentation at the Interdisciplinary Conference: Preparing \\ for a Sustainable Society, Toronto, 21-22 June, 1991
}

\begin{abstract}
$\underline{\text { Abstract }}$
A sustainable society at present world population levels is faced with many complex issues: curbing further human population growth, preventing nuclear, biological or chemical wars, soothing social and political tensions, fighting poverty, protecting the environment from poison and climatic change, coping with resource scarcity, and managing vulnerable ecosystems. Each one of the items in this list transcends our conventional disciplines. Considering further that all of the problems are connected makes it obvious that neither a scientifically illiterate public nor our professionals, traditionally trained in narrow disciplines, are capable of creating or maintaining a sustainable society.

Scientific and ordinary literacy of the general public is a desirable if not a necessary preparation for a sustainable society. Can it be achieved through our present educational means, or is it necessary for education to change? Today, the alphabet and grammar have become simple enough for all to learn how to read and write with a minor effort, and illiteracy in developed countries is now the exception rather than the rule. Unfortunately, this is not the case with scientific literacy. To learn science today is hard and time consuming. Our scientific and engineering knowledge is fragmented into many disciplines, and our curricula in these fields are cluttered with insignificant details. The frustrating information overload prevents most contemporaries from becoming scientifically literate, and it is difficult to get even the simplest of scientific truths to a wide public.

A new knowledge structure for the development of a unified science curriculum is presented in this paper. By using universal concepts and universally applicable algorithms of thinking, a knowledge core is presented which connects all the disciplines and avoids duplication. It is concluded that such a unified science reduces the quantity of information required for a broad view of existing knowledge, that the reduced effort in learning such a universal mental tool will motivate more students to think scientifically about broad issues, and that the professionals trained in transdisciplinary sciences will be able to see the "big picture" of the problems facing a sustainable society.
\end{abstract}

\section{Mankind's Dreams and How They Came Partially True}

As we look back in history, we see persistent desires, dreams of mankind. Some examples are: making gold, searching for perpetual motion, the fountain of youth, the stone of wisdom, clairvoyance, longevity, and peace.

Through the ages man was able to realize some of these goals, partially at least, and often in unexpected ways. Alchemists have turned into pharmacists, chemists, or even nuclear chemists. The astrologers are now astronomers or psychologists. The seven mile boots, the flying carpet, and the witches'-brooms are realized as fast cars, planes and rockets. The idol Siegfried is now Superman, but the dream of the wonder sword is a cruel reality in the form of ABC bombs. 


\section{APPROPRIATE CURRICULUM}

The 'Nümberger Trichter', the funnel of wisdom has been observed in the animal kingdom: ants are known to transfer information with a drop of fluid. A compact disk 'memory', glittering in rainbow colours, is our modern crystal bali. We may ask it many questions and get a knowledgeable answer. The semi-conductor chip is the stone of wisdom occurring in man's early dreams.

Paradise and the land of milk and honey, or in modern terms, peace and a sustainable society are still a far distance away. Longevity of the world population is increasing every year, but war, hunger, illness and early death persist in large parts of the world. These social and ecological goals cannot be reached with presently available knowledge tools.

Science and technology deserve credit for the realization of many of mankind's dreams, and of course, they are to be blamed for some of the failures and undesirable environmental effects of our achievements. For the purpose of realizing and maintaining a sustainable society science and technology, our mental and physical tools, need improvement in order to match the challenges.

\section{The Nature and the Challenges of a Sustainable Society}

A sustainable society is a large and complex system. Such big systems are slow in reacting to change and, therefore, their controlled operation demands long term planning. All of these features, the size, the complexity, and the long term planning represent challenges which tax the existing scientific and technical tools at our disposal.

As the description of the state and the function of complex systems requires observation or prediction of many variables, one may visualize each of these variables as a coordinate in an abstract, multidimensional problem space. Obviously, the higher the number of variables necessary to understand and predict the behaviour of a system, the greater is the challenge to sustain such a system in the long term.

The difficulty in solving multidimensional problems is aggravated by the fact that the variables usually depend on each other. The variation of one variable through the full range does not lead to the discovery of the right value of this variable for the solution of the problem unless all the others are simultaneously right too. The solution to a complex, multidimensional problem requires that the right position must be found simultaneously in all dimensions.

An illustration of the difficulty in solving multidimensional problems is given in the following examples:

The problem of guiding two pencils to meet at one point in space requires simultaneous observations in four dimensions. Guiding each pencil to the same point in space does not make a successful meeting; if the pencils are there at different times, we do not have a successful meeting. Holding a pencil in each hand we may easily observe their up down and left right position at a given time; however, we are lacking reliable information about the forward and backward position, and thus we experience difficulty in solving the problem of guiding the two pencils together.

Windsurfing is another example of a multidimensional problem. It requires right positions of the boat, the mast, the sail and the person, all relative to each other, and relative to the wind and the waves, and all that as a function of time. The novice experiences frustration when trying to acquire the skill by trial and error. Due to the multidimensional character of the activity, success will not be experienced even when most of the parameters are right, success only comes when all are right 


\section{APPROPRIATE CURRICULUM}

simultaneously. The difficulty is that the novice does not even notice when an individual variable is in the right position; there is no reward or feedback for placing a single parameter in the right position. Therefore, the beginner's 'stabbing in the dark' success is unlikely to happen in a many dimensional problem. A good instructor who teaches some of the right positions on the basis of his own experience reduces the dimensionality of the problem for the learner, and that is a great help.

It is important to recognize the full dimensionality of a problem. Whenever we do not recognize the full dimensionality of a problem, there are difficulties in finding a solution. A creature, familiar with two dimerisional spaces only, will find it difficult to understand why the two pencils don't meet, when both the space positions and the timing are right.

A sustainable society poses problems of many dimensions. For a global community to live in harmony with itself and with its environment requires an ability to cope with more complexity than we have experienced before. The challenge of a sustainable society is one of many dimensions. We consider three parts of the problem. The first challenge is to cope with the complexity: The big picture challenge', or 'The whole elephant challenge'. The second part has to do with timing, not missing the boat: 'The quick response challenge'. The third challenge is related to making use of collective intelligence in a democratic operation of the sustainable society: The wide access challenge'.

The current disciplinary structure of our knowledge is unfit to meet any of the challenges. The issues of war and peace, of social and economic development on a global scale, of protecting the oceans of the world and the rain forests, and the green land with all the life forms in them, and the issues raised by genetic engineering all transcend the existing disciplines of knowledge. Therefore knowledge in its present structure is not effective enough to meet the future challenge. Even if it were possible to get the 'big picture' of a sustainability issue from our dispersed disciplinary knowledge, it would be burdened with several handicaps. First, a representative from each discipline would have to be part of any decision making process, and we know from experience that too many cooks spoil the broth. Secondly, it would be difficult to get a quick response since the communication across the disciplinary boundaries requires translations of the science dialects, and that takes time. Thirdly, our scientific knowledge in its present form does not always conform with common sense, and often is not expressed in common language. Thus, it is the preserve of many leagues of specialists, inaccessible to the general public, and unfit to meet the wide access challenge.

Our success in meeting the challenges of a sustainable society depends on finding the right mental and physical tools which will enable us to recognize the whole problem, to respond quickly, and to utilize the collective intelligence of the global community.

\section{Universal Knowledge Tools}

"There is nothing more practical than a good theory"

Ludwig Boltzmann

Language is the most basic and transdisciplinary mental tool we have. Scientists and engineers use it, poets and businesspeople use it, philosophers and politicians use it. Language adapts to common sense and to mathematical rigour. Every human being acquires a language. The early versions of written language were difficult to learn; it took devoted scholars a lifetime effort to acquire the skills of reading and writing. Today, the alphabet and grammar have been structured in 


\section{APPROPRIATE CURRICULUM}

such simple form that most people learn how to read and write in their early childhood. The early versions of all tools are primitive.

The first counting tools were pictures of the objects to be counted. An improved counting tool uses token objects, fingers or pebbles which have no similarity with the objects to be counted apart from being distinct units. This property of being a distinct unit is observable in all disciplines, and it makes counting a truly transdisciplinary activity. The tools people use are improved as their culture progresses. The Roman numerals worked well for counting, but had restricted potential in the manipulation of counted numbers. The arabic numerals are excellent tools for counting and for arithmetic. Algebra introduces symbols to represent any number and streamlines the rules which regulate the manipulation of numbers. Today, mathematics offers a set of highly developed tools of thinking which are truly transdisciplinary. Mathematics is used in all branches of knowledge.

Computers and telecommunication devices are the equivalent physical tools corresponding to language and mathematics. These tools are also transdisciplinary in nature. After a primitive start with the adding machine, computers are now popular in all sectors of our society. They do not recognize the disciplinary boundaries.

Natural languages are transdisciplinary tools, but they are not universal tools since they have cultural boundaries. The reason for this confusion of tongues is the independent development of languages in many independent geographical areas of the World. At present we find some 150 complete languages in use around the Globe. The odd gap in one of the languages may be discovered in translations, but it is filled by coining new words, or by transferring a word from another language. The notion of 'fairness' for example, first developed in the English culture, is an accepted term in German. Vice versa, the German notion of 'Gemütlichkeit' has no adequate translation, and therefore, the German word is frequently used in English. With such minor repairs, each of the many current languages is fully capable of expressing anything that needs to be said in any discipline or culture.

And yet, with all this excess of languages, we do not have a tool for global communication. Professionals, today, are capable of communicating swiftly across some of the national boundaries. Most of them speak at least one foreign language. But a truly global communication instrument could be introduced by political action. The United Nations could designate one of the natural languages for it. Another option is to create a new, synthetic language for the purpose. Esperanto is an example for this option. With the global acceptance of just one of the languages a universal tool is created.

However, communication between the branches of knowledge, across the disciplinary boundaries, is more difficult. There is less overlap than in the common languages. Each discipline has developed its own conceptual structure, and ways of thinking, their own jargon, or 'branch dialect'. Translations between these are not only difficult, they may be impossible. There are many reasons for that. The branch dialects are special purpose devices. While some of the science dialects have the potential for a complete knowledge representation, most of the disciplines are fragments of a world view and not complete languages. Therefore we find less overlap, less common ground, and bigger gaps between them.

Is it possible to find a complete knowledge structure, a unified science theory which is a transdisciplinary, and potentially universal mental tool? Can a general science theory conform with the structure of natural language and common sense, and can it be conceptually rigorous so as to allow for the direct application of mathematical tools? In other words: can we discover a selfsimilarity in our highly complex 'knowledge fractal', can we find a simple generating formula for 


\section{APPROPRIATE CURRICULUM}

it? Or: Can we find a Copernican view of our knowledge universe, and discover the fixed points in it around which the rest of what we know revolves? Such a simplified yet universally applicable mental tool would be useful for meeting the big picture challenge, the quick response challenge, and the wide access challenge of a sustainable society.

A unified science theory is a desirable, if not necessary, tool for a sustainable society. It is for a responsible world citizen what the world map is to the globe trotter. The world map offers much less detail than a thousand regional maps. But it allows the traveler to gain the "big picture" fast, and is much simpler than the regional maps, and thus, it allows for easy access to information. Once the big picture is formed, the traveler needs to study the detailed maps only around the point of origin, and upon arrival at the destination. In a similar fashion a unified science simplifies the utilization of knowledge in a sustainable global community.

The importance of a powerful theory and the proper choice of the world view for a good education can be demonstrated by a historical example: the Copernican simplification of astronomy. Copernicus is not remembered for any spectacular discoveries of celestial phenomena. $\mathrm{He}$ is remembered and famous for introducing a changed, new state of the mind while looking at unchanged astronomical data. This change of mind is similar to the sudden switch in perception when we observe certain graphical patterns. For example, the identical pattern of steps we see going up or down, depending on the state of our mind. Space travel would be practically impossible without the improvement of Astronomy theory by Copernicus. The Ptolemean view of the world from a satellite is quite different from the Ptolemean world of an Earth based observer. Each spaceship would require its own astronomic data bank. The astronomic data would vary with the state of motion of the ship. And what happens when the spaceship starts wobbling in a nutational motion? And what is the equation of motion of a comet? Recording Ptolemean observations from a satellite would be a waste of effort - the data would be falsified by the astronaut moving away from the telescope. Ptolemean dynamics for a spaceship rendezvous would be a challenge for super computers due to the lack of a common and inertial reference frame. It is obviously better to collect the time invariable data from an inertial frame of reference resting in all the suns, and not in each individual spaceship.

\section{Appropriate Curriculum}

The purpose of searching for an appropriate curriculum is to assist the student in meeting the challenges of a complex world: the big picture challenge, the quick response challenge, and the wide access challenge.

What are the criteria for selecting appropriate curriculum items?

- Simplicity, to avoid information overload,

- Familiarity, to make use of common sense,

- Universality, to avoid duplication and to avoid tunnel vision, and

- Rigour, to allow for the utilization of mathematical tools.

What is the core of an appropriate curriculum in a unified science theory? It is a transdisciplinary knowledge structure based on the systematic use of universal concepts and universally applicable algorithms of thinking. The concepts and the thinking algorithm must be able to incorporate, with some adjustment, the concepts, the conceptual structure and the thought processes of all the diciplines.

How can such a simple, and common knowledge structure be discovered in what presently appears 


\section{APPROPRIATE CURRICULUM}

to be a diverse, and complex body of knowledge? Does it, by any chance, already exist in some of the many branches of science? Can it be found by combining and expanding existing branches of science? A transdisciplinary, unified science has been proposed as a combination of thermodynamics and accounting, enriched with some of Aristotle's brilliant, universal concepts [1].

Based on a unified science the following items are suggested for an appropriate curriculum:

\section{Substance}

Aristotle's universal notion of substance is useful in a unified science theory. Unfortunately the originally wide concept has experienced a change in meaning. Today's notion of substance is narrow, almost restricted in its meaning to chemical elements and compounds. In Aristotle's original definition the substance concept was meant to cover everything which exists. In a modern interpretation of that purpose it includes such existents as mass, energy, momentum, electric charge; elementary particles, atoms, molecules, solid objects, planets, stars; life organisms etc. Substances are identified by their space, time, and motion attributes. It is interesting to note that the classification of our knowledge in disciplines is largely determined by a classification of Aristotelian substances. Each class of Aristotelian substance is the object of study of one of the established disciplines. Chemistry, for example, deals with atoms and molecules, biology with organisms, astronomy with stars.

\section{Space. Time, and Motion.}

These concepts are universal. They are, in one way or another, relevant in every discipline of knowledge. The related topics reach from postal addresses to analytic geometry, from common dates to the simultaneity concept in special relativity, and from scheduling and time studies to kinematics of points and of continua.

\section{System. Accounting Entity. Gauss Container}

The thermodynamic notion of a 'system' is equivalent to the 'accounting entity' in the world of business. A universally applicable definition of the unit to which the accounting algorithm is applied is the 'Gauss container'. It is defined by an imagined closed surface in space. Whatever substance is inside that Gauss bubble belongs to the system, and what is outside does not belong. At first, such a system looks like a set. But, a Gauss container is not a set, it is a domain in real, physical space. It is a device which allows us to count, or account for the substances which are contained in it.

\section{State. Inventory. Change of State}

The state of a system, which is defined as a Gauss container, can be described by a list of contents, by an inventory of substances. The change of state can be described by a list of inventory changes. 


\section{APPROPRIATE CURRICULUM}

\section{Processes: Transfer and Production}

Only two processes leading to a change in the substance inventory of a Gauss container are logically possible. One is the production or destruction of a substance within the container, and the second is the transfer of a substance through the surface of the container.

\section{The Universal Equation of Change}

The change of the substance inventory in a Gauss container can be related to the processes of transfer and production by a universally valid 'balance equation' which, in transport theory or fluid dynamics is called the 'continuity equation'. The change DX of the amount of a substance X in a container is equal to the sum total of all transfers TX and all productions PX:

$$
\mathrm{DX}=\mathrm{TX}+\mathrm{PX} \text {. }
$$

This equation in a variety of forms can be applied to solve problems not only in accounting, but in many other disciplines [2]. Its use to develop a unified theory of classical physics is given in [3].

\section{Conclusion}

It is maintained in this paper that the 'big picture challenge', the 'quick response challenge', and the 'wide access challenge' of a sustainable society cannot be met by existing, discipline oriented education which is based on fragments of the whole body of knowledge. For historic reasons, the academic disciplines present the sector of knowledge covered by them in a discipline specific language, conceptual framework, and algorithm of thinking. The non-matching thought forms resist the unification of knowledge fragments, prevent quick responses to transdisciplinary problems, and do not allow public access to the whole body of knowledge.

The proposed new knowledge structure is substance accounting. It is universally applicable, and thus, it assists the student to meet the big picture challenge, and it helps to avoid duplication in the various branches of knowledge. The unified science curriculum proposed is simple, based on the familiar, common sense thought process of accounting, and it is scientifically rigorous, allowing for the full utilization of our mathematical tools and of computer power.

[1] H. Burkhardt, Substance Accounting: A Fundamental Knowledge Structure for All Disciplines, Proceedings of the Annual General Meeting of the Americal Society for Engineering Education, Toronto, June 1990.

[2] A.A. Bartlett, The Continuity Equation in Architecture and Planning. Abstract in the American Association for Physics Teacher's Bulletin: The Announcer, 21, 96,(1991)

[3] H. Burkhardt, System Physics: A Uniform Approach to the Branches of Classical Physics, Am. J. Phys., 55, 344 (1987) 